\title{
Circulating miR-375 as a novel prognostic marker for metastatic medullary thyroid cancer patients
}

\author{
Paola Romeo1, Carla Colombo2,3, Roberta Granata4, Giuseppina Calareso5, Ambra Vittoria Gualeni6, \\ Matteo Dugo7, Loris De Cecco7, Maria Grazia Rizzetti', Angela Zanframundo6, Antonella Aiello6, \\ Maria Luisa Carcangiu6, Annunziata Gloghini6, Stefano Ferrero8,9, Lisa Licitra4,10, Angela Greco', \\ Laura Fugazzola2,3*, Laura Deborah Locati4* and Maria Grazia Borrello1*

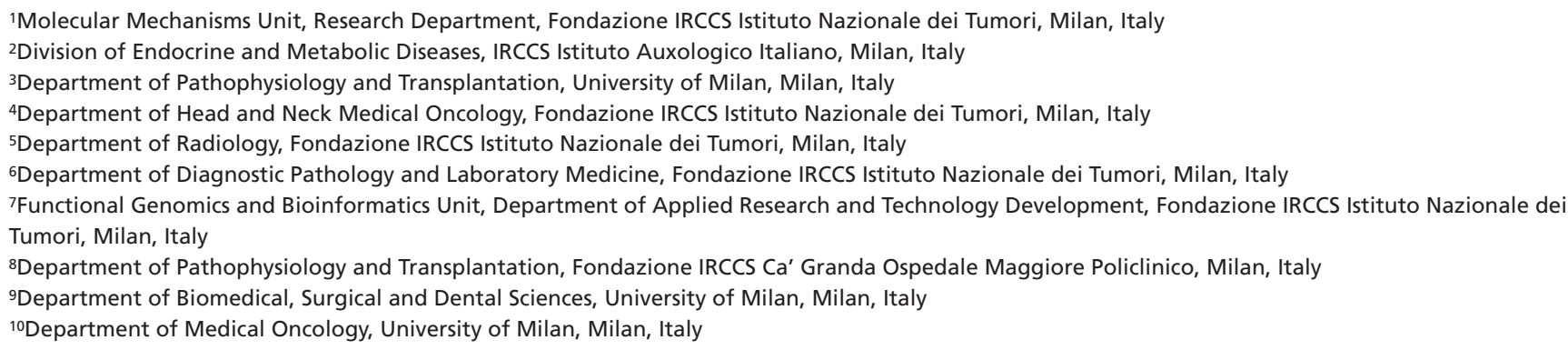

\section{Abstract}

This study aimed to identify circulating miRNAs as novel non-invasive biomarkers for prognosis and vandetanib response in advanced medullary thyroid cancer (MTC) patients. We prospectively recruited two independent cohorts of locally advanced/metastatic MTC patients including a subgroup of vandetanib-treated subjects: a discovery cohort $(n=20)$, including matched plasma/tissue samples $(n=17 / 20)$, and a validation cohort, yielding only plasma samples $(n=17)$. Plasma samples from healthy subjects $(n=36)$ and MTC patients in remission ( $n=9)$ were used as controls. MTC ( $n=17$ from 8 patients included in discovery cohort) and non-neoplastic thyroid specimens $(n=3)$ were assessed by microarray profiling to identify candidate circulating miRNAs. qRT-PCR and in situ hybridization were carried out to validate the expression and localization of a selected miRNA within tissues, and qRT-PCR was also performed to measure miRNA levels in plasma samples. By microarray analysis, we identified 51 miRNAs differentially expressed in MTC. The most overexpressed miR, miR-375, was highly expressed by $C$ cells compared to other thyroid cells, and more expressed in MTC than in reactive C-cell hyperplasia. MTC patients had significantly higher miR-375 plasma levels than healthy controls $(P<0.0001)$ and subjects in remission $(P=0.0004)$ as demonstrated by qRT-PCR analysis. miR-375 plasma levels were not predictive of vandetanib response, but, notably, high levels were associated with significantly reduced overall survival (HR 10.61, $P<0.0001)$ and were a strong prognostic factor of poor prognosis (HR 6.24, $P=0.00025$ ) in MTC patients. Overall, our results unveil plasma miR-375 as a promising prognostic marker for advanced MTC patients, to be validated in larger cohorts.

\section{Key Words}

- medullary thyroid cancer

- prognostic biomarker

- circulating miRNA

- miR-375

- metastases 


\section{Introduction}

Medullary thyroid cancer (MTC) is a rare neuroendocrine malignancy with an incidence rate of 0.21 cases per 100,000 person-years (Wells et al. 2015, Randle et al. 2017). MTC originates from the calcitonin-producing $\mathrm{C}$ cells that represent the minor thyroid cell population (less than $0.1 \%$ of the glandular mass). About $25 \%$ of MTC cases are inherited as familial syndrome (FMTC) or as a component of type 2 multiple endocrine neoplasia syndromes (MEN2A, MEN2B) due to germline-activating mutations in the RET proto-oncogene (Leboulleux et al. 2004). The remaining $75 \%$ of MTC occurs sporadically, with somatic $R E T$-activating mutations identified in $50 \%$ of cases, and somatic HRAS and KRAS mutations in $17-68 \%$ of RET-negative patients (Moura et al. 2011, Ciampi et al. 2012).

The 10-year overall survival is approximately 95\% in patients with intra-thyroid disease, decreasing to $75 \%$ and $40 \%$ in patients with regional disease and distant metastases, respectively (Roman et al. 2006). Local invasiveness and regional lymph node metastases are present in more than $75 \%$ of cases at diagnosis, while diffuse distant metastasization, mainly in the liver, lung and bones, is observed in $4-17 \%$ of patients (Moley \& DeBenedetti 1999, Hadoux et al. 2016). The standard surgical treatment, total thyroidectomy and neck dissection, leads to remission almost exclusively in patients with intra-thyroidal disease. Therefore, many patients require repeated interventions for persistent or recurrent MTC. The clinical course of these individuals is heterogeneous and difficult to predict: it varies from metastatic diffusion with rapid progression to death, to indolent/slow-growing disease that remains stable for long time or unexpectedly progresses even a decade after the first treatment.

After unsuccessful surgery, the therapeutic options are limited. Currently, locally advanced and metastatic MTC patients with progressive and/or symptomatic disease are candidates for systemic treatment with multitarget tyrosine kinase inhibitors (TKIs) as vandetanib or cabozantinib.

The monitoring of calcitonin (Ctn) and carcinoembryonic antigen (CEA) serum levels is a central tool in the follow-up of patients. The Ctn and CEA doubling times, when shorter than 6 months, are negative prognostic indicators of progressive disease. However, reliable estimates require repeated measurements over a minimum of 2 years (Wells et al. 2015). Furthermore, in patients treated with TKIs, fluctuation in these serum markers have been described regardless of tumor response (Kurzrock et al. 2013, Werner et al. 2015).

A better patients' prognostic assessment, able to differentiate indolent from aggressive MTC, could significantly improve the clinical management. In patients with progressive disease, the choice of the precise timing to start a systemic treatment, such as vandetanib or other TKIs, is crucial considering the side effects of these drugs and the life-long duration of therapy.

Moreover, there are no clinical neither biological data available to select patients who could benefit from TKI therapy. Thus, there is an urgent need to identify novel non-invasive biomarkers useful for prognosis stratification and to evaluate TKI therapy effectiveness in MTC patients.

In this scenario, circulating cell-free microRNAs (miRNAs) may provide a valuable resource. Nowadays, it is well established that miRNA deregulation is a hallmark of cancer and that distinct tumor types have a unique miRNA profile (Calin \& Croce 2006). Tumorderived miRNAs are present in the blood of cancer patients and may be useful, in patient management, as novel non-invasive biomarkers for diagnosis, prognosis and prediction of therapeutic response (Mitchell et al. 2008, Schwarzenbach et al. 2014). Nevertheless, miRNAs distinctive of MTC are still poorly characterized (Chu \& Lloyd 2016) and, above all, circulating miRNAs have never been analyzed in this tumor type. We hypothesized that miRNAs overexpressed in tumor tissue may be good candidate circulating biomarkers in the plasma of MTC patients, as their levels may be associated with specific clinicopathological characteristics, response to therapy and patient outcome.

The aims of this study were to explore, for the first time, the expression of tumor-specific miRNAs in MTC patients' plasma samples and to investigate their value as biomarker for prognosis and vandetanib response in persistent/recurrent MTC patients. To these purposes, we analyzed a miRNA overexpressed by MTC cells in the plasma of two cohorts of locally advanced or metastatic MTC patients, including a subgroup of patients treated with vandetanib.

\section{Materials and methods}

\section{Study subjects and study design}

A total of 37 MTC patients with persistent or recurrent metastatic disease, 9 non-metastatic MTC patients in remission and 36 healthy controls were enrolled 
at Fondazione IRCCS Istituto Nazionale Tumori and at Fondazione IRCCS Ca' Granda Ospedale Maggiore Policlinico between 2011 and 2015. The clinicopathological characteristics of the study population are detailed in Supplementary Tables 2 and 3 (see section on supplementary data given at the end of this article).

All MTC patients underwent total thyroidectomy associated in most cases with neck dissection and received a diagnosis of hereditary or sporadic MTC. Cases included persistent or recurrent MTC patients who had locally advanced/metastatic disease with at least one lesion measurable by standard imaging procedures. Subjects with a history of other malignant tumors were excluded. Systemic treatment and/or radiotherapy had to be discontinued at least 4 weeks prior to study entry. After enrolment, 25 patients with progressive (according to Response Evaluation Criteria in Solid Tumors (RECIST) 1.1 criteria (Eisenhauer et al. 2009)) and/or symptomatic disease received vandetanib. Treatment consisted of oncedaily oral vandetanib, administered at approved doses.

Patients in remission and healthy subjects were also enrolled as controls. Remission was defined as no structural evidence of disease (NED) and undetectable Ctn and CEA within the reference range during the follow-up (median follow-up from surgery: 65 months). The study was approved by the Independent Ethical Committees of Istituto Nazionale Tumori and $\mathrm{Ca}^{\prime}$ Granda Ospedale Maggiore Policlinico; each subject provided written informed consent upon recruitment.

Patients flow through the study is schematically represented in Fig. 1, whereas the number of samples used in each analysis is summarized in Supplementary Table 1. MTC patients were prospectively recruited in two independent cohorts: we first sequentially recruited 20 MTC patients in the 'discovery cohort' (MTC1), and subsequently, 17 MTC patients in the 'validation' cohort (MTC2) following the same inclusion and exclusion criteria. Healthy control subjects were selected to match MTC patients for gender and age distribution (HC1 $(n=19)$ and HC2 $(n=17))$.

Formalin-fixed paraffin-embedded (FFPE) surgical specimens were obtained from 17/20 MTC patients of the discovery cohort (MTC1 FFPE). Five non-neoplastic thyroid and four thyroid with diagnosis of C-cell hyperplasya (CCH) FFPE were collected for comparison.

Baseline blood samples were obtained from all the subjects at study entry (T0). One patient of the discovery cohort was subsequently excluded from the study of

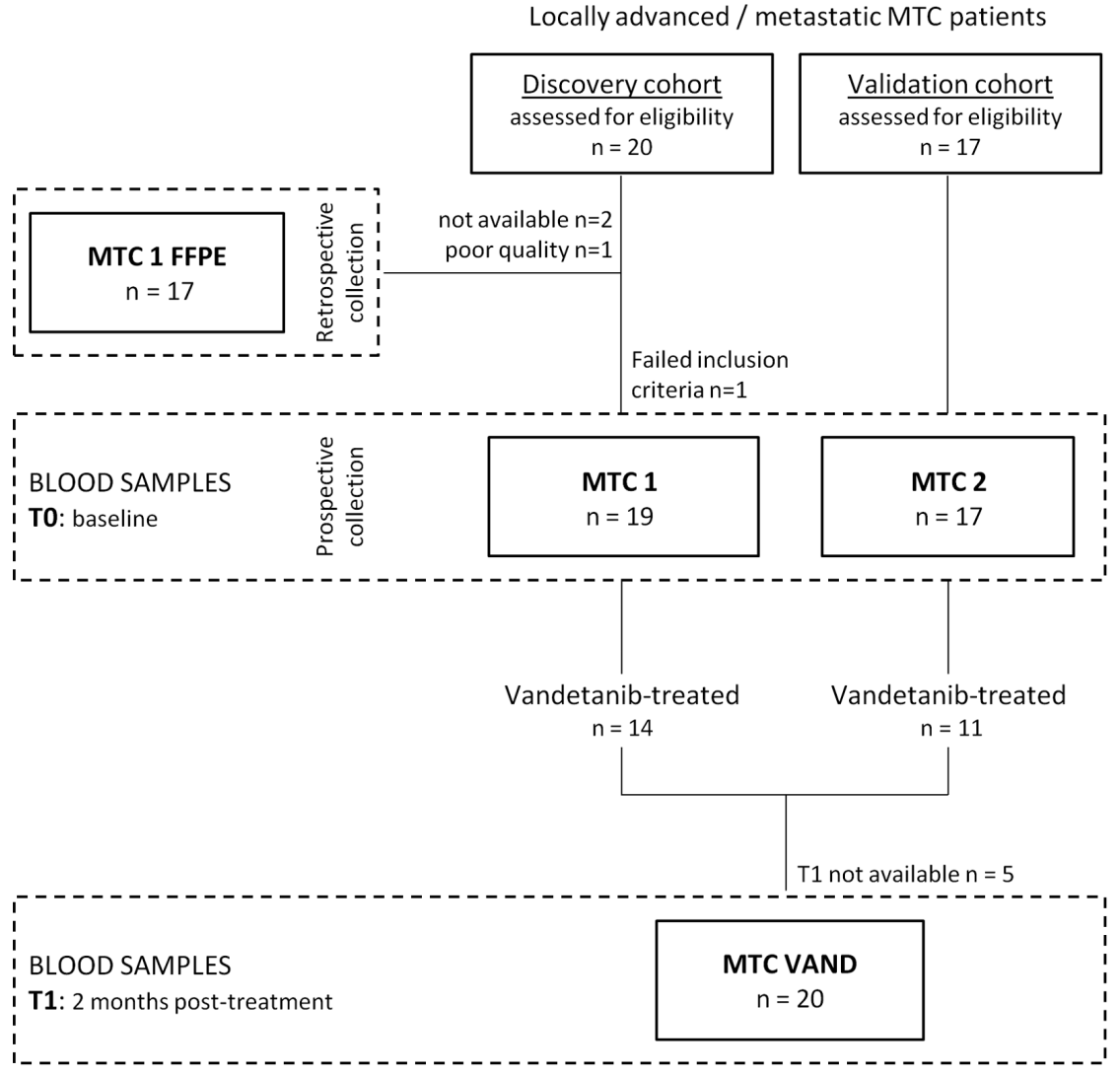

Figure 1

Flowchart of patients recruitment and samples collection 
circulating miRNAs for a concurrent pancreatic tumor diagnosed after the enrolment. For the subgroup of patients treated with vandetanib, paired blood samples were collected at study entry (T0) and 2 months after the start of vandetanib treatment (T1). To investigate the variations of candidate circulating miRNAs after treatment, we considered only 20 patients (MTC VAND), out of the 25 treated with vandetanib, as 5 cases were excluded due to the lack of the post-therapy blood sample.

Patients were followed for tumor progression with radiological imaging at regular intervals as for clinical practice. The clinical response to vandetanib was determined based on the RECIST 1.1 criteria (Eisenhauer et al. 2009) and the first evaluation of objective response was performed at a median time of 3 months (range 2-4 months) after the start of vandetanib. Overall survival (OS) was calculated from the date of blood withdrawal to the date of death or of the last follow-up.

\section{Tumor burden assessment}

Metastatic burden was estimated by a semi-quantitative method, and a score ranging from 0 to 100 was assigned to each persistent/recurrent MTC patient. An experienced radiologist (GC) reviewed images performed at study entry (total body TC and MRI for bone lesions) and calculated the total burden score for each patient. Five sites of disease were identified: bone, mediastinal nodes, liver and lung, that are the most common sites for distant metastases, plus one additional site called 'other' including neck and less frequent distant metastasis sites (e.g. kidney, brain, adrenal gland). According to the definition of 'target lesion' described in RECIST 1.1 (Eisenhauer et al. 2009), all target and non-target lesions were analyzed in each site, and a score ranging from 0 to 20 was assigned: 0 for no lesions, 5 for non-target lesions only, 10 for up to two target lesions, 15 for up to four target lesions and 20 for $\geq$ five target lesions. The tumor burden score was calculated as the sum of the scores for each site.

\section{Biological sample collection and nucleic acid extraction}

Twenty-six FFPE specimens, comprising primary tumors and/or nodal metastases, were obtained from 17 MTC patients. Five non-neoplastic thyroid FFPE specimens were obtained from subjects undergone thyroidectomy for benign thyroid nodular hyperplasia. Four thyroid FFPE specimens with diagnosis of $\mathrm{CCH}$ were obtained from patients operated for benign nodular goiter. CCHs were classified as 'reactive', namely 'reactive C-cell proliferation not associated with MTC' (Perry et al. 1996). Prior to nucleic acids extraction, hematoxylin- and eosinstained and calcitonin-immunostained tissue sections were reviewed by an experienced pathologist (MLC), and the areas of interest were manually microdissected. DNA was extracted by proteinase $\mathrm{K}$ digestion in PCR buffer at $56^{\circ} \mathrm{C}$ overnight; total RNA was extracted using the miRNeasy FFPE kit (Qiagen) and its quality was assessed by Bioanalyzer (Agilent).

A total of 101 blood samples from cases and controls were analyzed. From each subject, $10 \mathrm{~mL}$ of blood were collected in an EDTA vacuum tube and processed within $2 \mathrm{~h}$ by centrifugation at $2000 \mathrm{~g}$ for $10 \mathrm{~min}$ at room temperature. Plasma fraction was transferred to RNasefree tubes and stored at $-80^{\circ} \mathrm{C}$ until RNA extraction. A lipemia-independent hemolysis score (HS) was calculated for each plasma samples as previously described (Appierto et al. 2014). Total RNA, was extracted from plasma using the miRNeasy Kit (Qiagen) essentially as previously reported (Callari et al. 2013). Briefly, plasma samples were thawed on ice and centrifuged at $2000 \boldsymbol{g}$ for $10 \mathrm{~min}$ at $4^{\circ} \mathrm{C}$. Subsequently, $350 \mu \mathrm{L}$ of plasma were mixed with $1300 \mu \mathrm{L}$ of Qiazol Lysis Reagent (Qiagen) plus $1.25 \mu \mathrm{g} / \mathrm{mL}$ of MS2 bacteriophage RNA carrier (Roche) and $0.15 \mathrm{fmol}$ of UniSp6 exogenous synthetic RNA (Exiqon).

Genomic DNA was extracted from peripheral blood by the commercial QIAamp DNA Blood Mini Kit (Qiagen) following the manufacturer's instructions.

\section{RET and RAS mutational status analysis}

Germline and somatic RET gene status was assessed for the occurrence of mutations in exons $8,10,11,13,14,15$ and 16 , while somatic $R A S$ gene mutations were evaluated in exons 1, 2 and 3 (H-RAS) and exons 2, 3 and 4 (K-RAS). $200 \mathrm{ng}$ of DNA were amplified and directly sequenced as previously reported (Hofstra et al. 1996, Moura et al. 2011, Boichard et al. 2012).

\section{miRNA microarray profiling}

miRNA microarray profiling was performed on 8 MTC cases, selected as representative of the entire discovery cohort for stages (TNM) and genetic lesions, among those that had both primary tumor and lymph node metastasis specimens available. Three non-neoplastic thyroid specimens, with the highest RNA quality, were included for comparison. RNA was labeled and processed according to the manufacturer's recommended protocol, 
and miRNA expression was analyzed using SurePrint G3 Human miRNA 8x60K microarrays (Agilent Technologies), as previously described (De Cecco et al. 2017). Primary data were collected using Agilent's Feature Extraction software, v10.7 (Agilent Technologies). The data discussed in this publication have been deposited in NCBI's Gene Expression Omnibus (Edgar et al. 2002) and are accessible through GEO series accession number GSE97070 (https:// ncbi.nlm.nih.gov/geo/query/acc.cgi?acc=GSE97070).

\section{miRNA bright field in situ hybridization}

miRNA bright field in situ hybridization (ISH) studies were performed on $4 \mathrm{CCH}$ and 5 MTC FFPE samples (from the discovery cohort), as previously described (Gualeni et al. 2015) by using double-DIG-LNA probes (Exiqon). HsamiR-21 and scramble-miR probes were used respectively as positive and negative controls to ensure adequate sample quality and specificity of staining. Positive signals appeared as brown dots usually localized in the cytoplasm. Signals were quantified using a scoring system from 0 to 16 obtained by multiplying the number of dots/cell $(0=$ no signal, 1=1-3 dots/cell, 2=4-6 dots/cell, 3=7-9 dots/cell and $4=>10$ dots/cell) with the percentage of positive cells $(1=0-24 \%, 2=25-49 \%, 3=50-75 \%$ and $4=>75 \%)$.

\section{Real-time quantitative reverse transcription PCR}

miRNA expression was quantified by two-step real-time quantitative reverse transcription-PCR (qRT-PCR). For tissues miRNA, 50ng of RNA was reverse transcribed by the TaqMan MicroRNA Reverse Transcription Kit (Applied Biosystems). Quantification was performed by TaqMan miRNA assays (Applied Biosystems). RNU6B small nucleolar RNA, already reported as endogenous control in MTC (Abraham et al. 2011, Mian et al. 2012), was used for data normalization. For plasmatic miRNAs, qRT-PCR was performed by the miRCURY LNA Universal RT microRNA PCR system (Exiqon) according to the manufacturer's instruction. Synthetic RNA UniSp6 was used for normalization. All qRT-PCR were performed in triplicate on the ABI PRISM 7900HT Real-Time PCR System. Data were analyzed with the Sequence Detection System 2.4 and the RQ Manager 1.2.1 software, using the $2^{-\Delta \Delta C t}$ method with a confidence interval (CI) set at $95 \%$.

\section{Statistical analysis}

For microarray analysis, miRNAs differentially expressed between MTC and non-neoplastic thyroid samples were identified using the limma $\mathrm{R}$ package (Phipson et al. 2016). Multiple-testing correction was performed using the Benjamini-Hochberg false discovery rate (FDR). miRNAs with FDR $<0.05$ and absolute fold-change $\geq 2$ were considered differentially expressed.

Statistical differences between qRT-PCR expression values and differences in patients clinicopathologic characteristics between the two case lists were evaluated by the Mann-Whitney $U$ test, Wilcoxon test or $\chi^{2}$ test as appropriate. Correlation between miRNA expression and tumor burden or HS was evaluated using the Spearman correlation coefficient. Receiver-operating characteristic (ROC) curves and area under the curve (AUC) were used to assess miR-375 ability to discriminate MTC patients from healthy control subjects or remission patients. The Youden index method (Ruopp et al. 2008) was applied to identify the optimal miRNA expression cut-off value. Survival analyses were performed by the Kaplan-Meier method and the differences in OS were assessed by the log-rank test. The Cox proportional hazard model was applied for univariate and multivariate analysis. For multivariate analysis, only significant variables from univariate analysis were considered. Association between categorical variables was assessed by Fisher's exact test.

All $P$ values were two sided, and $P<0.05$ was considered statistically significant. Statistical analyses were performed using Graphpad Prism version 5 and $\mathrm{R}$ software with related Bioconductor packages.

\section{Results}

\section{Patient characteristics}

Patient clinicopathological features, including RET/RAS mutational status, are detailed in Supplementary Table 2 and summarized in Table 1. According to germline RET genotyping, 33 patients had sporadic MTC, 2 patients had MEN2B and 2 patients had isolated familial MTC. Among patients with sporadic MTC, M918T was the most common RET mutation, being present in 16 out of the 24 cases with known RET/RAS mutational status. The clinicopathological characteristics did not differ significantly between the two cohorts of MTC patients analyzed for circulating miRNAs (MTC1 $n=19$ and MTC2 $n=17)$. MTC patients were matched for sex and age with the respective group of healthy controls (HC1 $n=19$ and HC2 $n=17)$. For both cases and controls, the mean age at study entry was 55 years (range: 19-82 years for MTC and $14-86$ years for $\mathrm{HC}$ ), and $58 \%$ of the subjects were males (21 males and 15 females for both MTC and HC). 
Table 1 Clinicopathological characteristics of the medullary thyroid cancer (MTC) patients.

\begin{tabular}{|c|c|c|c|}
\hline Patients characteristics & MTC1 $(n=19)$ & MTC2 $(n=17)$ & P-value a \\
\hline Sex, $n(\%)$ & & & 0.47 \\
\hline Male & $10(53)$ & $11(65)$ & \\
\hline Female & $9(47)$ & $6(35)$ & \\
\hline \multicolumn{4}{|l|}{ Age, mean (range) years } \\
\hline Age at diagnosis & $50(28-68)$ & $48(8-82)$ & 0.96 \\
\hline Age at TO & $57(32-77)$ & $54(19-82)$ & 0.80 \\
\hline $\begin{array}{l}\text { Primary tumor } \\
\quad \text { classification, } n(\%)\end{array}$ & & & 0.96 \\
\hline $\mathrm{T} 1 / \mathrm{T} 2$ & $5(28)$ & $4(29)$ & \\
\hline T3/T4 & $13(72)$ & $10(71)$ & \\
\hline $\mathrm{Na}$ & 1 & 3 & \\
\hline $\begin{array}{l}\text { Lymph node status at } \\
\text { diagnosis, } n(\%)\end{array}$ & & & 0.30 \\
\hline $\mathrm{NX}$ & $1(6)$ & $2(14)$ & \\
\hline NO & $0(0)$ & $1(7)$ & \\
\hline $\mathrm{N} 1 \mathrm{a} / \mathrm{b}$ & $17(94)$ & $11(79)$ & \\
\hline $\mathrm{Na}$ & 1 & 3 & \\
\hline Sporadic/genetic forms & & & 0.08 \\
\hline Sporadic & $19(100)$ & $13(76)$ & \\
\hline MEN2B & $0(0)$ & $2(12)$ & \\
\hline Familial MTC & $0(0)$ & $2(12)$ & \\
\hline $\begin{array}{l}\text { Metas.tatic disease at T0, } \\
\quad n(\%)\end{array}$ & & & 0.59 \\
\hline 1 metastatic site & $6(32)$ & $4(24)$ & \\
\hline$\geq 2$ metastatic sites & $13(68)$ & $13(76)$ & \\
\hline Extent of disease, $n(\%)$ & & & 0.12 \\
\hline $\begin{array}{l}\text { Only locoregional } \\
\text { disease }\end{array}$ & $1(5)$ & $4(24)$ & \\
\hline $\begin{array}{l}\text { Distant metastatic } \\
\text { disease }\end{array}$ & $18(95)$ & $13(76)$ & \\
\hline Site of disease, $n(\%)$ & & & 0.74 \\
\hline Bone & $12(63)$ & $9(53)$ & \\
\hline Liver & $10(53)$ & $7(41)$ & \\
\hline Lung & $6(32)$ & $9(53)$ & \\
\hline Mediastinum & $11(58)$ & $11(65)$ & \\
\hline Other & $10(53)$ & $13(76)$ & \\
\hline \multicolumn{4}{|l|}{$\begin{array}{l}\text { Follow-up, median } \\
\text { (range) months }\end{array}$} \\
\hline FU from surgery & $80(22-243)$ & 75 (18-203) & 0.72 \\
\hline FU from TO & $30(3-59)$ & $18(3-63)$ & 0.20 \\
\hline $\begin{array}{l}\text { Status at last follow-up, } \\
n(\%)\end{array}$ & & & 0.09 \\
\hline DWD & $11(58)$ & $5(29)$ & \\
\hline AED & $8(42)$ & $12(71)$ & \\
\hline
\end{tabular}

Tumors were classified and staged according to the thyroid malignancy World Health Organization classification Organization classification (Hedinger et al. 1988) and the 6th edition of TNM staging (Compton et al. 2012). T0, time corresponding to baseline blood sample collection. aClinicopathologic characteristics of the two case lists of MTC patients were compared by $\chi^{2}$ test or Mann-Whitney $U$ test, as appropriate. AED, alive with evidence of disease; DWD, dead with disease; FU, follow-up; MTC, medullary thyroid cancer; na, data not available.

According to the TNM classification, 23 out of the 32 patients with available clinical information (72\%) had advanced tumors (T3-T4) and 28/32 (88\%) had lymph node metastases at the initial surgery. At study entry, 5/36 patients (14\%) had only locoregional disease, whereas 31/36 (86\%) had distant metastases. Twenty-six/36 MTC patients $(72 \%)$ had multiple metastatic lesions involving different organs. The most common distant sites of metastases were mediastinum (61\%), bone (58\%), liver (47\%) and lung (42\%). At data cut-off (September 2016), the median duration of follow-up was 25 months, 16/36 patients (44\%) had died of disease and 20/36 (56\%) were alive with evidence of disease.

\section{miRNA expression in MTC tissue samples}

To identify candidate circulating miRNAs in MTC patients, we first performed a miRNA microarray analysis of 8 primary tumors and 9 paired nodal metastases (6 synchronous and 3 metachronous), as cases representative of the entire discovery cohort, in comparison with 3 non-neoplastic thyroid tissues. We identified 51 miRNAs differentially expressed in MTCs compared to non-neoplastic thyroids (Fig. 2A). In MTCs, 27 miRNAs were overexpressed (the top ranking three were miR-375, miR-10a-5p and miR-4465) and 24 were underexpressed (the top ranking three were miR-30a-5p, miR-130a-3p and let-7i-5p). Almost all primary and metastatic lesions from the same patients clustered together, with the exception of two metachronous metastases. Moreover, we did not find any miRNA significantly differentially expressed between nodal metastases and primary tumors, showing they share a common miRNA expression profile. miR-375 was the most overexpressed miRNA: its expression was 22.49-fold higher in MTC specimens than that in nonneoplastic tissues, with similar levels between primary tumors and nodal metastases, either synchronous or metachronous (Fig. 2B). Expression analysis in all the available MTCs and non-neoplastic specimens by qRT-PCR validated the microarray results, as shown in Fig. 2C. miR-375 relative expression was consistently higher in all the analyzed tumors than that in non-neoplastic thyroid specimens $(P=0.001)$. The proper normal control for MTC is difficult to obtain since, in non-neoplastic thyroid, $\mathrm{C}$ cells are poorly represented and interspersed throughout the gland. To overcome this problem, we analyzed the miR-375-specific expression and localization by ISH analysis in reactive $\mathrm{CCH}$ samples, as enriched source of non-neoplastic C cells, and in MTC cases. We observed that miR-375 signal was markedly prevalent in the cytoplasm of the Ctn-positive hyperplastic C cells and MTC cells (Fig. 2D). Its expression was consistently higher in tumor (mean score: 15 ) than in hyperplastic C cells (mean score: 8 ). Conversely, stromal and non-neoplastic follicular cells 

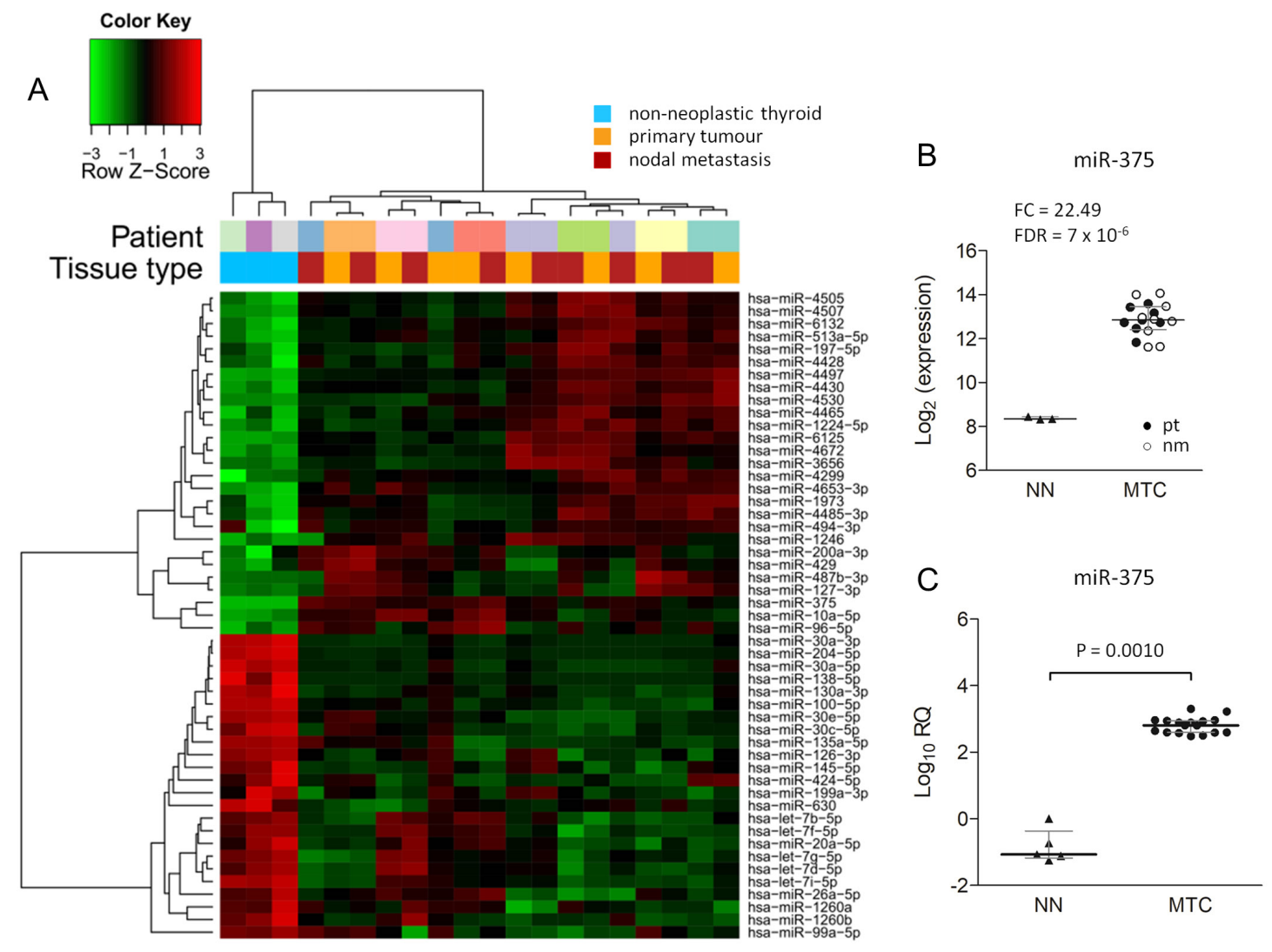

D $H \& E$ Ctn miR-375
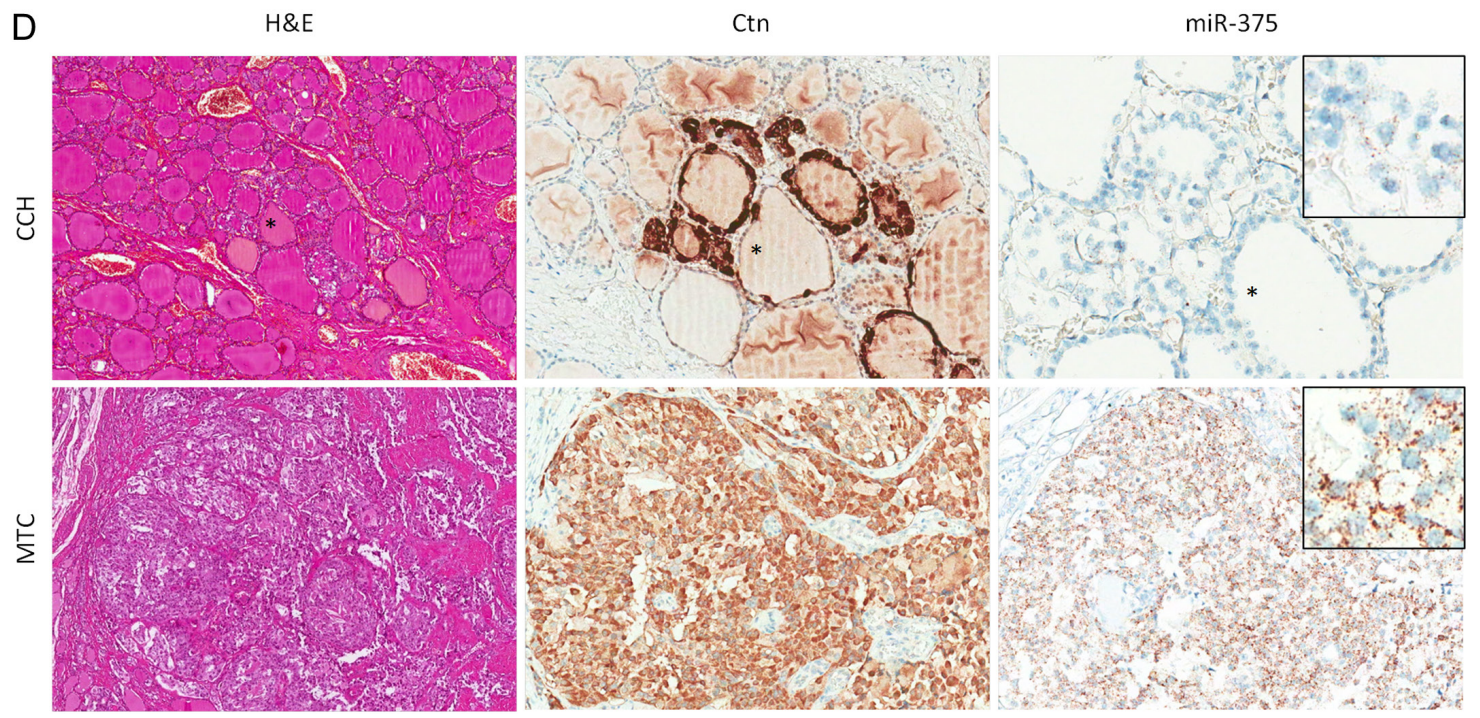

\section{Figure 2}

miRNA expression in MTC tissue samples. (A) heatmap representing color-coded expression levels of the 51 miRNAs differentially expressed in MTC primary tumours $(n=8)$ and matched nodal metastases $(n=9)$ compared to non-neoplastic thyroids $(n=3)$. (B) Scatter dot plots represent miR-375 microarray intensity expression values in tumor (MTC; pt, primary tumor; nm, nodal metastasis) and non-neoplastic thyroid samples (NN). (C) Scatter dot plots represent miR-375 qRT-PCR relative quantification values (RQ) in MTCs (MTC, $n=17$ ) and non-neoplastic thyroid samples (NN, $n=5$ ). MTC and NN samples used in microarray profiling were also included in the qRT-PCR analysis. Data represent the mean values from two independent PCR experiments. In scatter dot plots horizontal lines indicate median values and error bars indicate the interquartile range. Statistical significance was evaluated by Mann-Whitney $U$ test. (D) Hematoxylin and eosin stain (H\&E), immunohistochemistry for calcitonin (Ctn) and bright field ISH for miR-375 (miR-375) in one example of CCH and MTC FFPE specimens. In the cases shown, miR-375 expression is strong in neoplastic cells (score 16) and moderate in hyperplastic $\mathrm{C}$ cells (score 6). Original magnification: H\&E, 4x; Ctn, 10x; miR-375 ISH, 20x for CCH, 10x for MTC; inset 40x. Images are acquired by Aperio Scanscope whole-slide imaging. 
displayed negative or very low miR-375 expression (Supplementary Fig. 1A and B).

These results indicate that miR-375 represents a promising candidate to be investigated in the blood of MTC patients.

\section{miR-375 expression in MTC plasma samples}

miR-375 levels were analyzed in the plasma samples obtained at baseline from 19 MTC patients of the discovery cohort (MTC1) and in 19 sex- and age-matched healthy controls (HC1) by qRT-PCR. miR-375 was detected in all samples. No significant correlation was observed between the plasma levels of miR-375 and the degree of hemolysis of the specimens, ensuring that miR-375 levels were not influenced by possible blood cell disruption occurring during sample preparation (Supplementary Fig. 2A and B). Interestingly, miR-375 mean relative expression level was significantly higher in MTC patients than in the control group ( $P=0.0003$; Fig. $3 \mathrm{~A})$. This result was validated in an independent cohort of 17 MTC patients (MTC2), compared with 17 sex- and age-matched healthy controls (HC2, $P=0.0119$; Fig. 3B). The ROC curve analyses showed that miR-375 levels were moderately accurate in discriminating patients from controls, with AUC values of 0.85 (95\% CI: 0.72-0.98) and 0.75 (95\% CI: 0.58-0.93) for the discovery and the validation cohort, respectively (Fig. 3C and D).

Despite plasma miR-375 levels being very low in the control subjects, we hypothesized that normal C cells of the healthy thyroid could concur in miR-375 secretion. Therefore, we examined the differences in miR-375 expression levels between the whole group of metastatic MTC patients (MTC1 and MTC2 analyzed together) and a group of MTC patients in remission (MTC NED), both groups without C cells from normal thyroid as possible source of circulating miR-375. The latter had slightly, though not statistically significant, lower levels of miR-375 than control subjects, and significantly lower levels of miR-375 than persistent/recurrent MTC patients $(P=0.0004$; Fig. 3E). Importantly, miR-375 levels were able to discriminate patients in remission from those with persistent/recurrent MTC, with an AUC value of 0.88 (95\% CI: 0.78-0.99). According to the optimal cut-off value of 0.04 persistent/recurrent MTC patients were identified with sensitivity and specificity of $86.1 \%$ and $88.9 \%$, respectively (Fig. 3F).
These results demonstrate that plasma miR-375 levels are significantly higher in MTC patients than in healthy subjects and patients in remission.

\section{Association of plasma miR-375 levels with clinicopathological characteristics and overall survival in MTC patients}

We investigated the possible association between miR-375 levels and patients' clinicopathological features. Notably miR-375 levels were significantly higher in individuals with distant metastases than in those with locoregional disease only ( $P=0.0019$; Fig. $4 \mathrm{~A})$. Moreover, after subgrouping patients by the tumor burden score $(<50$ and $\geq 50)$, we observed that those with a widespread disease had the highest levels of miR-375 $(P=0.0197$; Fig. 4B) and that its expression was significantly correlated with the tumor burden $(r=0.6341, P<0.0001$; Fig. 4C). Male patients displayed higher miR-375 expression than females $(P=0.0061$; Fig. 4D), while no difference was observed by sex among healthy controls (Supplementary Fig. 3A). No significant association was found with patients' age at diagnosis or mutational status (Supplementary Fig. 3B and C).

As the parameters associated with higher miR-375 levels may influence the disease course, we investigated whether plasma levels of miR-375 were associated with the patients' outcome. The prognostic significance of miR-375 was determined, within the subgroup of patients with distant metastasis, by Kaplan-Meier survival analysis (Fig. 4E). The relative miR-375 expression levels were categorized, according to the median, into high ( $\geq$ median) and low ( $<$ median). This analysis revealed that patients with higher levels of miR-375 had a striking and significantly worse OS $(P<0.0001 ; \mathrm{HR}=10.61 ; 95 \%$ CI: 3.809-29.53). Median follow-up duration was 36 months (14-63 months) and 19 months (3-43 months) for the group of patients with low and high levels of plasma miR-375, respectively. In addition, univariate survival analysis showed that poor prognosis was associated only with male sex $(P=0.02715)$, tumor burden $(P=0.0749)$ and high plasmatic levels of miR-375 ( $P=0.00004$; Table 2). Patient outcome was not associated with age at diagnosis, RET/RAS mutational status or vandetanib treatment. Notably, by multivariate survival analysis, only high levels of miR-375, but not male sex nor tumor burden, maintained the prognostic significance of worse outcome $(\mathrm{HR}=5.52 ; 95 \% \mathrm{CI}$ : $1.98-15.41 ; P=0.00108$; Table 2). 
A

$A$ $\operatorname{miR}-375$

$\operatorname{miR}-375$
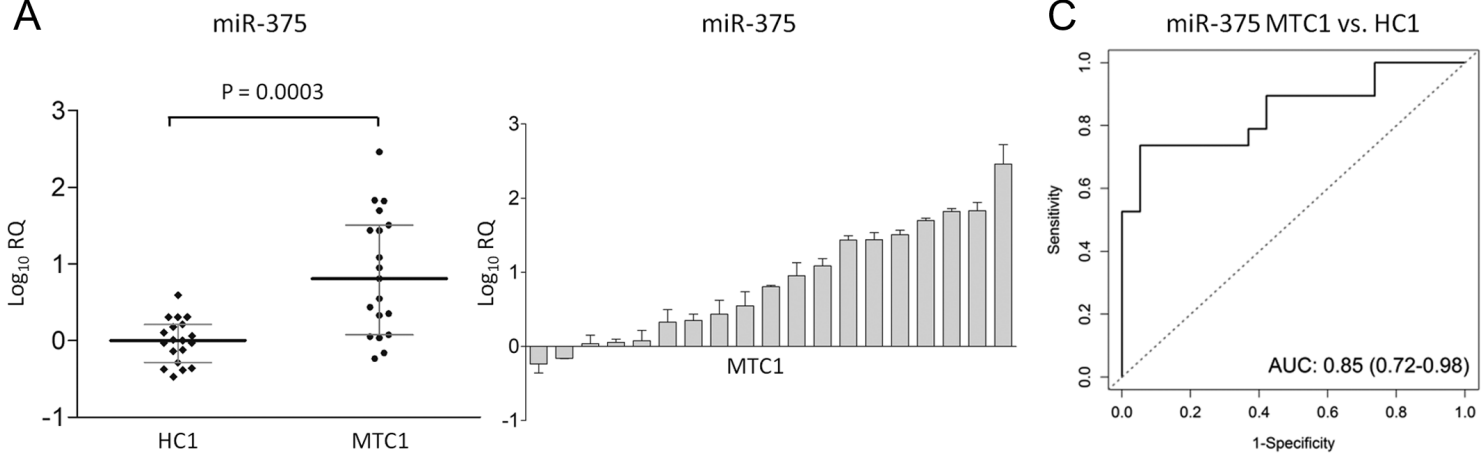

B

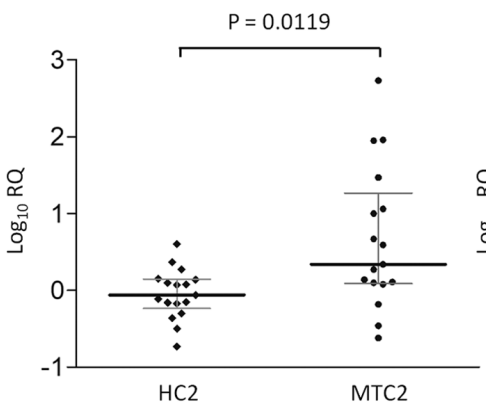

miR-375
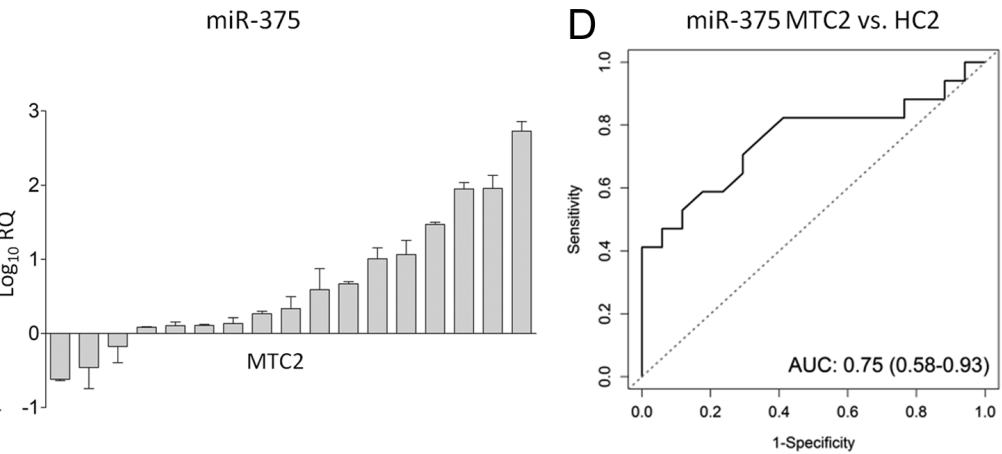

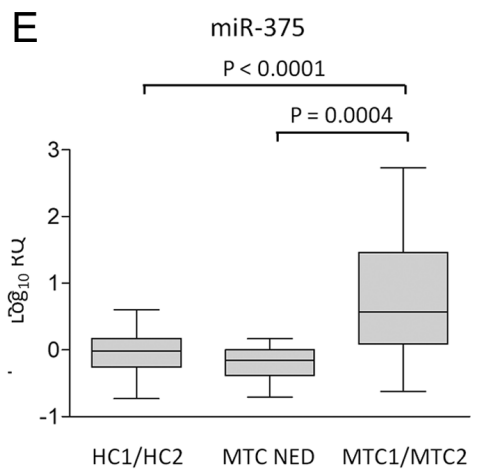

F

MTC1/MTC2vs. MTCNED

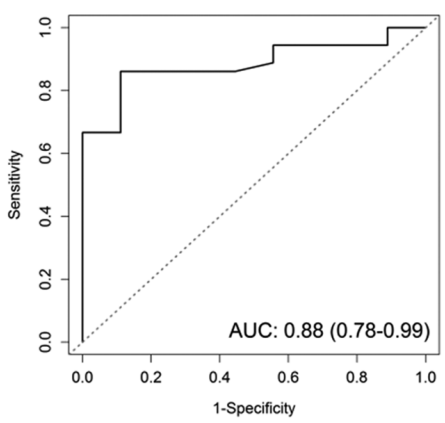

\section{Figure 3}

miR-375 expression in plasma samples. (A and B) Scatter dot plots (left panels) represent miR-375 qRT-PCR relative quantification values (RQ) in plasma samples of MTC patients in the discovery and in the validation cohorts (MTC1, $n=19 ;$ MTC2, $n=17$ ) and matched healthy controls (HC1, $n=19 ; \mathrm{HC} 2$ $n=17$ ). Horizontal lines indicate median RQ values, error bars indicate the interquartile range. Histograms (right panels) show the distribution of miR-375 RQ in MTC1 and MTC2 plasma samples. Data represent mean values+s.D. from two independent PCR experiments. (C and D) ROC curve analysis for plasma miR-375 plotted to discriminate MTC patients from HC subjects in the two cohorts. (E) Box plots represent plasma miR-375 RQ values in HC1-HC2 $(n=36)$ healthy controls, MTC patients in remission (MTC NED $n=9)$ and in MTC1-MTC2 combined cohorts $(n=36)$. Boxes represent the interquartile range, horizontal lines indicate median values and error bars indicate maximum and minimum values. Statistical significance was evaluated by Mann-Whitney $U$ test. (F) ROC curve analysis for plasma miR-375 plotted to discriminate MTC patients in persistence/recurrence (MTC) from MTC patients in remission (MTC NED).

\section{Plasma miR-375 analysis in patients treated with vandetanib}

Variations of plasma miR-375 levels were investigated in a subset of 20 patients treated with vandetanib (Supplementary Table 4). At the first objective response evaluation after the start of vandetanib, 9 patients (45\%) achieved a partial response (PR), 10 patients (50\%) had stable disease (SD) and 1 patient (5\%) was in progression (PD). RETM918T-positive patients had a statistically significant $(P=0.016)$ higher response rate to vandetanib (PR in 66.7\%) compared with 
A

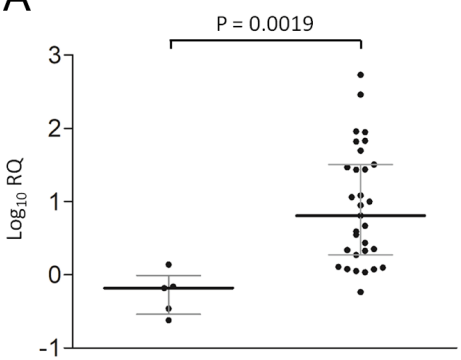

MTC LR

$\mathrm{D}$

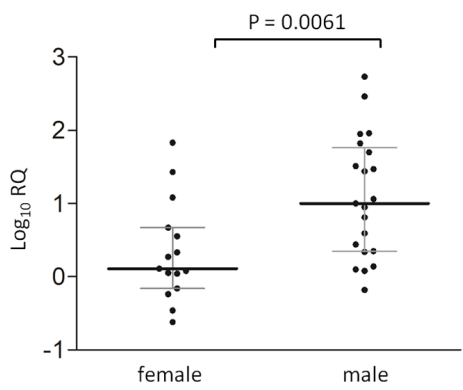

B

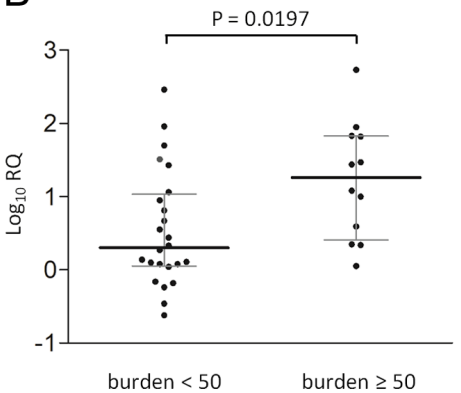

E

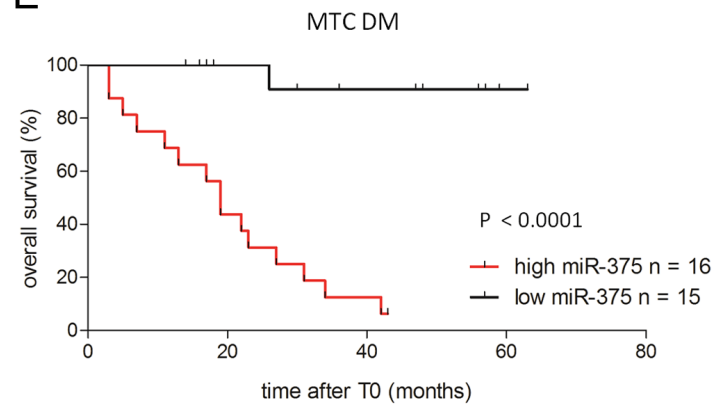

C

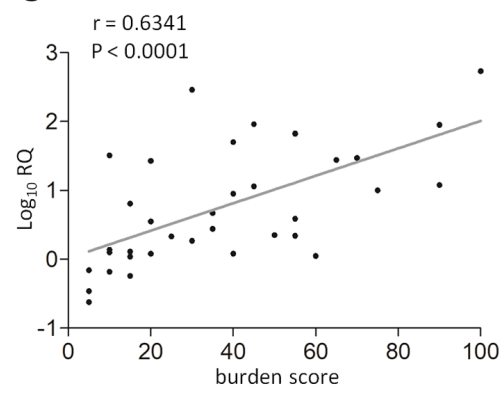

$\mathrm{F}$

MTC VAND

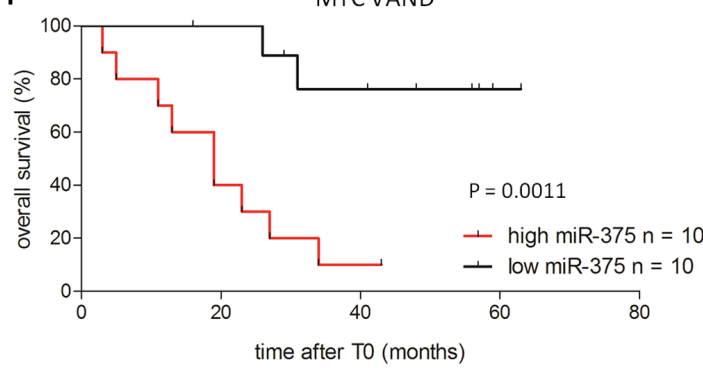

Figure 4

Association of plasma miR-375 levels with clinicopathological characteristics and overall survival in MTC patients. Scatter dot plots represent plasma miR-375 relative quantification values (RQ) (A) in MTC patients with locoregional disease only (MTC LR $n=5$ ) vs MTC patients with distant metastatic disease (MTC DM $n=31$ ) and (B) in MTC patients divided on the basis of tumor burden score (burden score $<50 n=24$ and $\geq 50 n=12$ ). (C) Scatter plot show the positive correlation between miR-375 RQ values and patients' tumor burden score defined by Spearman correlation analysis. (D) Plasma miR-375 levels in female $(n=15)$ and male $(n=21)$ MTC patients. In all scatter dot plots, horizontal lines indicate median RQ values and error bars indicate the interquartile range. Statistical significance was evaluated by Mann-Whitney $U$ test. (E and F) Kaplan-Meier overall survival analysis in MTC patients with distant metastasis (MTC DM in E, $n=31$ ) and in the subgroup of patients treated with vandetanib (MTC VAND in F, $n=20$ ) categorized, according to the median, into patients with high and low miR-375 plasma levels. Survival curves were compared using log-rank test.

other patients (PR in 0\%). A match-paired analysis revealed that the levels of miR-375 were not significantly different before and 2 months after the start of vandetanib, even when the analysis was performed separately in the group of patients that achieved a PR or had SD (Supplementary Fig. 4A and B). Although not statistically significant, patients with PR to vandetanib displayed a higher miR-375 level reduction from pre-therapy to post-therapy than patients with $\mathrm{SD}$. Indeed, mean RQ values were unchanged from T0 to $\mathrm{T} 1$ in patients with SD and were 3.4-fold reduced after treatment in patients that achieved a PR. Published by Bioscientifica Ltd.
C) 2018 Society for Endocrinology Printed in Great Britain
Remarkably, high levels of miR-375 were significantly associated with reduced OS also in the subgroup of vandetanib-treated patients $\quad(\mathrm{HR}=8.309 ; \quad 95 \% \quad \mathrm{CI}$ : 2.341-29.50; $P=0.0011$; Fig. 4F).

\section{Discussion}

In this study, by using microarray profiling of tumor tissues to drive the discovery of disease-specific circulating miRNA, we identified miR-375 as a candidate prognostic biomarker for metastatic MTC patients. 
Table 2 Univariate and multivariate Cox proportional hazard analysis for outcome in MTC patients with distant metastasis.

\begin{tabular}{l} 
Variables \\
\hline Plasmatic miR-375 levels \\
Age at diagnosis \\
Tumor burden score \\
Sex (male vs female) \\
Mutational status (RETM918T vs other) \\
Vandetanib treatment
\end{tabular}

\begin{tabular}{l}
\multicolumn{1}{c}{ Univariat } \\
\hline \multicolumn{1}{c}{$\mathrm{HR}(95 \% \mathrm{Cl})$} \\
\hline $7.03(2.79-17.75)$ \\
$1.01(0.97-1.05)$ \\
$1.03(1.01-1.06)$ \\
$5.49(1.21-24.84)$ \\
$0.41(0.14-1.20)$ \\
$1.29(0.40-4.13)$ \\
\hline
\end{tabular}

\begin{tabular}{cc} 
\\
\hline 0.00004 \\
0.56277 \\
0.00749 \\
0.02715 \\
0.10448 \\
0.66538 \\
\hline
\end{tabular}

\begin{tabular}{|c|c|}
\hline \multicolumn{2}{|c|}{ Multivariate } \\
\hline $\mathrm{HR}(95 \% \mathrm{Cl})$ & $P$ \\
\hline $5.52(1.98-15.41)$ & 0.00108 \\
\hline- & - \\
\hline $1.009(0.98-1.034)$ & 0.48271 \\
\hline $2.82(0.56-14.09)$ & 0.20644 \\
\hline- & - \\
\hline- & - \\
\hline
\end{tabular}

Univariate and multivariate analyses were performed for the 26 MTC patients with distant metastases that have all the considered clinicopathological data available. miR-375, age at diagnosis and tumor burden score were considered as continuous variables, whereas, sex, mutational status and vandetanib treatment as categorical variables. Only the variables that were statistically significant in univariate were considered for the multivariate analysis.

$\mathrm{Cl}$, confidence interval; $\mathrm{HR}$, hazard ratio.

There are few studies on miRNA expression in MTC, mostly focused on a limited group of miRNAs, analyzed by qRT-PCR (Mian et al. 2012, Duan et al. 2014, Gundara et al. 2014, Pennelli et al. 2015, Spitschak et al. 2017). Arraybased miRNA profiling in MTC was firstly performed in 2008 (Nikiforova et al. 2008) in different thyroid tumors, including 2 MTC cases, and only recently in larger cohorts of MTCs (Hudson et al. 2013, Lassalle et al. 2016). In this study, we performed a miRNA microarray profiling to identify miRNAs overexpressed in both primary tumors and metastases in a cohort of advanced patients (stage III and IV) to be challenged as circulating biomarkers in plasma derived from the same patients. We defined a signature of miRNAs differentially expressed in MTC compared to non-neoplastic tissue, that included miRNAs already recognized in MTC as miR-375, mir-10a, miR-127-3p, miR-138-5p and miR-130a-3p, together with a novel set of miRNAs previously unreported for this disease.

We demonstrated that nodal metastases and primary tumors had the same global miRNA profile, in agreement with previous data (Gundara et al. 2014). Although another recent study (Santarpia et al. 2013) identified a list of ten miRNA associated with metastatic MTC, we did not validate their signature in our caselist; we hypothesize that this can be either due to the use of different array platforms or due to the different patient selection criteria (all stage III and IV in our caselist).

Microarray data showed that miR-375 was the most upregulated miRNA in MTC primary tumors and nodal metastases, either synchronous or metachronous. While the overexpression of this miRNA has already been reported in MTC (Mian et al. 2012, Hudson et al. 2013, Lassalle et al. 2016), we provide, for the first time, more insights regarding the specific thyroidal miR-375 expression at the single-cell level by ISH analysis. This analysis allowed us to perform the more appropriate comparison with the normal counterpart of MTC, the benign C cells derived from reactive $\mathrm{CCH}$. We have coherently demonstrated that miR-375 is poorly expressed in normal follicular cells and, of notice, also in stromal cells of the tumor microenvironment. At variance, hyperplastic C cells express miR-375, though at lower levels than neoplastic $\mathrm{C}$ cells. Our results indicate that the high expression of miR-375 is associated with the C-cell lineage within the thyroid gland and suggest that its deregulation could be involved in MTC tumourigenesis. Indeed, miR-375 was already identified as aberrantly expressed in multiple types of cancer including other neuroendocrine tumors (Nishikawa et al. 2011, Miller et al. 2016), and overexpressed, in particular, in breast and prostate cancer (Yan et al. 2014).

Although the origin of circulating miRNAs is still controversial, previous reports demonstrate that miRNAs overexpressed in tumor tissues may be detected in plasma and correlate with patient clinicopathological characteristics and prognosis (Schwarzenbach et al. 2014). Circulating miRNAs display many advantages as biomarkers: they can be obtained with minimally invasive procedures, are easily quantified and have remarkable stability in the blood (Mitchell et al. 2008). We identified miR-375 as the best candidate to be quantified in the plasma of metastatic patients: (i) it was the most overexpressed miRNA in MTC and it was also abundantly expressed in metastases, supposed to be the source of circulating miRNAs in persistent/recurrent patients after thyroidectomy; (ii) it was poorly expressed in stromal cells within the tumor, a potential confounding source of circulating miRNAs; (iii) the circulating levels of miR-375 were not affected by the degree of hemolysis of the sample, and it was not mentioned among the miRNAs profiled in different peripheral blood cells (Duttagupta et al. 2011, Pritchard et al. 2012). In addition, miR-375 
expression levels in tumor have been recently correlated with clinical-pathological features and outcome of MTC patients (Galuppini et al. 2017), higher miR-375 expression being linked to a worst patient' outcome.

Importantly, we demonstrate that plasmatic miR-375 levels can discriminate with good sensitivity and specificity MTC patients from age- and gender-matched healthy controls and from MTC patients in remission. This latter group, being individual without thyroid as advanced MTC patients of our cohorts are, represents a further control group useful to investigate if normal C cells of the thyroid gland may in part contribute to the levels of plasma miR-375. We observed that patients in remission had slightly lower miR-375 levels than healthy controls, but the difference is not statistically significant suggesting that the contribution of normal $\mathrm{C}$ cells to the levels of plasma miR-375 is minimum.

We performed plasma miR-375 analysis by qRT-PCR using a synthetic spike-in RNA as normalizer. Although a general consensus about an optimal normalization strategy for circulating miRNA is still lacking, exogenous spike-in RNAs have already been exploited as normalisers in absence of a defined optimal endogenous miRNA control (Toiyama et al. 2013, Schwarzenbach et al. 2015).

We showed, in two independent cohorts, that circulating miR-375 levels are higher in metastatic MTC patients compared to controls and that a significant increase is observed in patients with advanced disease. Indeed, higher plasma miR-375 levels are associated with the presence of distant metastases and are directly correlated with the tumor burden, confirming the tumor origin of this miRNA. Circulating miR-375 levels were higher in male compared to female patients. Since this difference was not observed in matched healthy controls, we speculated that it is probably linked to a worse clinical course or to a more advanced disease in male patients, as previously reported (Saad et al. 1984, Pazaitou-Panayiotou et al. 2014).

Ctn and CEA doubling time are considered strong prognostic indicators of MTC progression and death (Barbet et al. 2005, Laure et al. 2008), though they display some critical issues. Indeed, the determination of Ctn and CEA doubling time is obtained by serial measurements to be performed over an extended period of time. Moreover, nearly all patients treated with RET inhibitors experienced an initial decline in serum Ctn or CEA and transient fluctuations that did not reflect only responsiveness to treatment, being due to the direct effect on Ctn and CEA transcription and secretion (Keno-Stuart et al. 2007, Kurzrock et al. 2013, Postel-Vinay et al. 2013, Werner et al. 2015).
Our data indicate that plasmatic miR-375 levels are significantly associated with patient outcome. In particular, among subjects with distant metastases, a strikingly reduced OS was found in those with higher miR-375 levels $\quad(H R=10.61 ; 95 \%$ CI: 3.809-29.53; $P<0.0001)$. Although tumor burden results are significantly associated to prognosis of advanced MTC patients (HR 1.03; 95\% CI 1.01-1.06; $P=0.00749$ ), plasmatic miR-375 shows a far better prognostic value (HR 7.03; 95\% CI: $2.79-17.75 ; P=0.00004)$ and is also significant in multivariate analysis.

Notably, 15/16 patients deceased at the time of data cut-off had miR-375 levels above the threshold fixed on the median value, and these high miR-375 levels were detected in plasma even 42 months before the patient's death. In contrast, only 1/15 patients still alive at the last follow-up had high miR-375 levels. Importantly, multivariate survival analysis showed that high miR-375 levels are a strong prognostic marker of poor outcome in patients with distant metastases $(\mathrm{HR}=5.52 ; 95 \% \mathrm{CI}$ : $1.98-15.41 ; P=0.00108)$.

Moreover, we demonstrate that the prognostic value of miR-375 is also maintained in the subgroup of patients treated with vandetanib. In addition, a higher miR-375 reduction from pre- to post-therapy was observed in patients who achieved PR vs those with SD. Although the statistical significance has not been reached, likely due to the limited number of patients analyzed, the trend observed suggests to consider miR-375 also as a possible marker of response to treatments in future studies. To assess this point, further studies involving a larger cohort of patients and blood samples at multiple time-points are needed. In the future, it would be also interesting to investigate the possible application of miR-375 in MTC diagnosis and follow-up, by testing its levels in patients before thyroidectomy and during the course of the disease, or to distinguish aggressiveness at presentation, e.g. metastatic vs non-metastatic MTC at diagnosis; novel cohorts of MTC patients need to be recruited for these purposes.

Since no predictive markers are currently available to select which patients with advanced MTC could benefit from systemic TKIs, radiological progression of disease according to RECIST 1.1 criteria remains the only factor of selection. We could speculate that in those patients with a low tumor burden along with a low miR-375 levels, systemic treatments could be delayed, favoring the employment of locoregional treatments. On the contrary, in those patients with high levels of miR-375, TKIs therapy should be promptly started in light of the worse prognosis. 
Our exploratory biomarker study points, for the first time, at circulating miR-375 as a novel independent prognostic marker for metastatic MTC patients. The main limitation of this study is the limited number of patients involved, which is mostly due to the low frequency of MTC.

Although validation of these results in a larger cohort is necessary, plasma miR-375 analysis seems a promising prognostic tool, as it may be evaluated, also in patients undergoing TKIs, by a minimally invasive, easy and costeffective procedure thus ultimately leading to better clinical decision on the management of metastatic MTC patients.

\section{Supplementary data}

This is linked to the online version of the paper at https://doi.org/10.1530/ ERC-17-0389.

\section{Declaration of interest}

L Licitra is a consultant/advisory board member for AstraZeneca, Bayer, Boehringer, Bristol-Myers Squibb, Debiopharm, Eisai, Merck-Serono, MSD, Novartis, Roche and Sobi; reports receiving research funds, through her institution, from AstraZeneca, Boehringer, Eisai, Merck-Serono, MSD, Novartis and Roche; and reports receiving travel reimbursement from Bayer, Debiopharm, Merck-Serono, and Sobi.; L D Locati received honoraria from EISAI. P Romeo received 1 year fellowship from Astra Zeneca. No potential conflicts of interest were disclosed by the other authors.

\section{Funding}

This work was partially supported by fellowship and grant from AstraZeneca.

\section{Acknowledgments}

The authors thank Dr Daniele Morelli for his assistance with data collection, and Dr Paola Tiberio for her methodologies advice. We acknowledge the contribution of Functional Genomic and Bioinformatics Core Facility of Fondazione IRCCS Istituto Nazionale dei Tumori for miRNA microarray experiments.

\section{References}

Abraham D, Jackson N, Gundara JS, Zhao J, Gill AJ, Delbridge L, Robinson BG \& Sidhu SB 2011 MicroRNA profiling of sporadic and hereditary medullary thyroid cancer identifies predictors of nodal metastasis, prognosis, and potential therapeutic targets. Clinical Cancer Research 17 4772-4781. (https://doi.org/10.1158/1078-0432. CCR-11-0242)

Appierto V, Callari M, Cavadini E, Morelli D, Daidone MG \& Tiberio P 2014 A lipemia-independent NanoDrop(R)-based score to identify hemolysis in plasma and serum samples. Bioanalysis 6 1215-1226. (https://doi.org/10.4155/bio.13.344)

Barbet J, Campion L, Kraeber-Bodere F \& Chatal JF 2005 Prognostic impact of serum calcitonin and carcinoembryonic antigen doublingtimes in patients with medullary thyroid carcinoma. Journal of
Clinical Endocrinology and Metabolism 90 6077-6084. (https://doi. org/10.1210/jc.2005-0044)

Boichard A, Croux L, Al GA, Broutin S, Dupuy C, Leboulleux S, Schlumberger M, Bidart JM \& Lacroix L 2012 Somatic RAS mutations occur in a large proportion of sporadic RET-negative medullary thyroid carcinomas and extend to a previously unidentified exon. Journal of Clinical Endocrinology and Metabolism 97 E2031-E2035. (https://doi.org/10.1210/jc.2012-2092)

Calin GA \& Croce CM 2006 MicroRNA signatures in human cancers. Nature Reviews Cancer 6 857-866. (https://doi.org/10.1038/nrc1997)

Callari M, Tiberio P, De Cecco L, Cavadini E, Dugo M, Ghimenti C, Daidone MG, Canevari S \& Appierto V 2013 Feasibility of circulating miRNA microarray analysis from archival plasma samples. Anaytical Biochemistry 437 123-125. (https://doi.org/10.1016/j.ab.2013.03.002)

Chu YH \& Lloyd RV 2016 Medullary thyroid carcinoma: recent advances including microRNA expression. Endocrine Pathology 27 312-324. (https://doi.org/10.1007/s12022-016-9449-0)

Ciampi R, Mian C, Fugazzola L, Cosci B, Romei C, Barollo S, Cirello V, Bottici V, Marconcini G, Pelizzo MR, et al. 2012 Evidence of a low prevalence of RAS mutations in a large medullary thyroid cancer series. Thyroid 23 50-57. (https://doi.org/10.1089/thy.2012.0207)

Compton CC, Byrd DR, Garcia-Aguilar J, Kurup P, Kurtzman SH, Olawaiye A \& Washington MK 2012 TNM classification of malignant tumors. In AJCC Cancer Staging Handbook. pp113-120. New York, NY, USA: Springer Verlag.

De Cecco L, Giannoccaro M, Marchesi E, Bossi P, Favales F, Locati LD, Licitra L, Pilotti S \& Canevari S 2017 Integrative miRNA-gene expression analysis enables refinement of associated biology and prediction of response to cetuximab in head and neck squamous cell cancer. Genes 8 35. (https://doi.org/10.3390/genes8010035)

Duan L, Hao X, Liu Z, Zhang Y \& Zhang G 2014 MiR-129-5p is downregulated and involved in the growth, apoptosis and migration of medullary thyroid carcinoma cells through targeting RET. FEBS Letters 588 1644-1651. (https://doi.org/10.1016/j.febslet.2014.03.002)

Duttagupta R, Jiang R, Gollub J, Getts RC \& Jones KW 2011 Impact of cellular miRNAs on circulating miRNA biomarker signatures. PLoS ONE 6 e20769. (https://doi.org/10.1371/journal.pone.0020769)

Edgar R, Domrachev M \& Lash AE 2002 Gene expression omnibus: NCBI gene expression and hybridization array data repository. Nucleic Acids Research 30 207-210. (https://doi.org/10.1093/ nar/30.1.207)

Eisenhauer EA, Therasse P, Bogaerts J, Schwartz LH, Sargent D, Ford R, Dancey J, Arbuck S, Gwyther S, Mooney M, et al. 2009 New response evaluation criteria in solid tumours: revised RECIST guideline (version 1.1). European Journal of Cancer 45 228-247. (https://doi. org/10.1016/j.ejca.2008.10.026)

Galuppini F, Bertazza L, Barollo S, Cavedon E, Rugge M, Guzzardo V, Sacchi D, Watutantrige-Fernando S, Vianello F, Mian C, et al. 2017 MiR-375 and YAP1 expression profiling in medullary thyroid carcinoma and their correlation with clinical-pathological features and outcome. Virchows Archiv 471 651-658. (https://doi.org/10.1007/ s00428-017-2227-7)

Gualeni AV, Volpi CC, Carbone A \& Gloghini A 2015 A novel semiautomated in situ hybridisation protocol for microRNA detection in paraffin embedded tissue sections. Journal of Clinical Pathology 68 661-664. (https://doi.org/10.1136/jclinpath-2015-203005)

Gundara JS, Zhao JT, Gill AJ, Clifton-Bligh R, Robinson BG, Delbridge L \& Sidhu SB 2014 Nodal metastasis microRNA expression correlates with the primary tumour in MTC. ANZ Journal of Surgery $\mathbf{8 4}$ 235-239. (https://doi.org/10.1111/j.1445-2197.2012.06291.x)

Hadoux J, Pacini F, Tuttle RM \& Schlumberger M 2016 Management of advanced medullary thyroid cancer. Lancet Diabetes Endocrinology 4 64-71. (https://doi.org/10.1016/S2213-8587(15)00337-X)

Hedinger C, Williams ED \& Sobin LH 1988 Histological Typing of Thyroid Tumours. WHO Second Edition. Berlin, Heidelberg, Germany: Springer-Verlag. (c) 2018 Society for Endocrinology Published by Bioscientifica Ltd. Printed in Great Britain 
Hofstra RM, Sijmons RH, Stelwagen T, Stulp RP, Kousseff BG, Lips CJ, Steijlen PM, Van Voorst Vader PC \& Buys CH 1996 RET mutation screening in familial cutaneous lichen amyloidosis and in skin amyloidosis associated with multiple endocrine neoplasia. Journal of Investigative Dermatology 107 215-218. (https://doi.org/10.1111/15231747.ep12329651)

Hudson J, Duncavage E, Tamburrino A, Salerno P, Xi L, Raffeld M, Moley J \& Chernock RD 2013 Overexpression of miR-10a and miR375 and downregulation of YAP1 in medullary thyroid carcinoma. Experimental and Molecular Pathology 95 62-67. (https://doi. org/10.1016/j.yexmp.2013.05.001)

Keno-Stuart N, Croyle M, Knauf JA, Malaguarnera R, Vitagliano D, Santoro M, Stephan C, Grosios K, Wartmann M, Cozens R, et al. 2007 The RET kinase inhibitor NVP-AST487 blocks growth and calcitonin gene expression through distinct mechanisms in medullary thyroid cancer cells. Cancer Research 67 6956-6964. (https://doi.org/10.1158/0008-5472.CAN-06-4605)

Kurzrock R, Atkins J, Wheler J, Fu S, Naing A, Busaidy N, Hong D \& Sherman S 2013 Tumor marker and measurement fluctuations may not reflect treatment efficacy in patients with medullary thyroid carcinoma on long-term RET inhibitor therapy. Annals of Oncology 24 56-2261.

Lassalle S, Zangari J, Popa A, Ilie M, Hofman V, Long E, Patey M, Tissier F, Belleannee G, Trouette H, et al. 2016 MicroRNA-375/ SEC23A as biomarkers of the in vitro efficacy of vandetanib. Oncotarget 7 30461-30478. (https://doi.org/10.18632/ oncotarget.8458)

Laure GA, Al GA, Auperin A, Leboulleux S, Chehboun A, Troalen F, Dromain C, Lumbroso J, Baudin E \& Schlumberger M 2008 Progression of medullary thyroid carcinoma: assessment with calcitonin and carcinoembryonic antigen doubling times. European Journal of Endocrinology 158 239-246. (https://doi.org/10.1530/EJE07-0667)

Leboulleux S, Baudin E, Travagli JP \& Schlumberger M 2004 Medullary thyroid carcinoma. Clinical Endocrinology 61 299-310. (https://doi. org/10.1111/j.1365-2265.2004.02037.x)

Mian C, Pennelli G, Fassan M, Balistreri M, Barollo S, Cavedon E, Galuppini F, Pizzi M, Vianello F, Pelizzo MR, et al. 2012 MicroRNA profiles in familial and sporadic medullary thyroid carcinoma: preliminary relationships with RET status and outcome. Thyroid 22 890-896. (https://doi.org/10.1089/thy.2012.0045)

Miller HC, Frampton AE, Malczewska A, Ottaviani S, Stronach EA, Flora R, Kaemmerer D, Schwach G, Pfragner R, Faiz O, et al. 2016 MicroRNAs associated with small bowel neuroendocrine tumours and their metastases. Endocrine-Related Cancer 23 711-726. (https:// doi.org/10.1530/ERC-16-0044)

Mitchell PS, Parkin RK, Kroh EM, Fritz BR, Wyman SK, PogosovaAgadjanyan EL, Peterson A, Noteboom J, O’Briant KC, Allen A, et al. 2008 Circulating microRNAs as stable blood-based markers for cancer detection. PNAS 105 10513-10518. (https://doi.org/10.1073/ pnas.0804549105)

Moley JF \& DeBenedetti MK 1999 Patterns of nodal metastases in palpable medullary thyroid carcinoma: recommendations for extent of node dissection. Annals of Surgery 229 880-887. (https://doi. org/10.1097/00000658-199906000-00016)

Moura MM, Cavaco BM, Pinto AE \& Leite V 2011 High prevalence of RAS mutations in RET-negative sporadic medullary thyroid carcinomas. Journal of Clinical Endocrinology and Metabolism 96 E863-E868. (https://doi.org/10.1210/jc.2010-1921)

Nikiforova MN, Tseng GC, Steward D, Diorio D \& Nikiforov YE 2008 MicroRNA expression profiling of thyroid tumors: biological significance and diagnostic utility. Journal of Clinical Endocrinology and Metabolism 93 1600-1608. (https://doi.org/10.1210/jc.20072696)

Nishikawa E, Osada H, Okazaki Y, Arima C, Tomida S, Tatematsu Y, Taguchi A, Shimada Y, Yanagisawa K, Yatabe Y, et al. 2011 miR-375 is activated by ASH1 and inhibits YAP1 in a lineage-dependent manner in lung cancer. Cancer Research 71 6165-6173. (https://doi. org/10.1158/0008-5472.CAN-11-1020)

Pazaitou-Panayiotou K, Chrisoulidou A, Mandanas S, Tziomalos K, Doumala E \& Patakiouta F 2014 Predictive factors that influence the course of medullary thyroid carcinoma. International Journal of Clinical Oncology 19 445-451. (https://doi.org/10.1007/s10147-0130588-8)

Pennelli G, Galuppini F, Barollo S, Cavedon E, Bertazza L, Fassan M, Guzzardo V, Pelizzo MR, Rugge M \& Mian C 2015 The PDCD4/miR21 pathway in medullary thyroid carcinoma. Human Pathology 46 50-57. (https://doi.org/10.1016/j.humpath.2014.09.006)

Perry A, Molberg K \& Albores-Saavedra J 1996 Physiologic versus neoplastic C-cell hyperplasia of the thyroid: separation of distinct histologic and biologic entities. Cancer 77 750-756. (https://doi. org/10.1002/(SICI)1097-0142(19960215)77:4<750::AIDCNCR22>3.0.CO;2-Z)

Phipson B, Lee S, Majewski IJ, Alexander WS \& Smyth GK 2016 Robust hyperparameter estimation protects against hypervariable genes and improves power to detect differential expression. Annals of Applied Statistics 10 946-963. (https://doi.org/10.1214/16-AOAS920)

Postel-Vinay S, Schlumberger M \& Soria JC 2013 Tumour markers fluctuations in patients with medullary thyroid carcinoma receiving long-term RET inhibitor therapy: ordinary lapping or alarming waves foreshadowing disease progression? Annals of Oncology 24 2201-2204. (https://doi.org/10.1093/annonc/mdt331)

Pritchard CC, Kroh E, Wood B, Arroyo JD, Dougherty KJ, Miyaji MM, Tait JF \& Tewari M 2012 Blood cell origin of circulating microRNAs: a cautionary note for cancer biomarker studies. Cancer Prevention Research 5 492-497. (https://doi.org/10.1158/1940-6207.CAPR-110370)

Randle RW, Balentine CJ, Leverson GE, Havlena JA, Sippel RS, Schneider DF \& Pitt SC 2017 Trends in the presentation, treatment, and survival of patients with medullary thyroid cancer over the past 30 years. Surgery 161 137-146. (https://doi.org/10.1016/j. surg.2016.04.053)

Roman S, Lin R \& Sosa JA 2006 Prognosis of medullary thyroid carcinoma: demographic, clinical, and pathologic predictors of survival in 1252 cases. Cancer 107 2134-2142. (https://doi. org/10.1002/cncr.22244)

Ruopp MD, Perkins NJ, Whitcomb BW \& Schisterman EF 2008 Youden index and optimal cut-point estimated from observations affected by a lower limit of detection. Biometrical Journal 50 419-430. (https:// doi.org/10.1002/bimj.200710415)

Saad MF, Ordonez NG, Rashid RK, Guido JJ, Hill CS Jr, Hickey RC \& Samaan NA 1984 Medullary carcinoma of the thyroid. A study of the clinical features and prognostic factors in 161 patients. Medicine 63 319-342. (https://doi.org/10.1097/00005792198411000-00001)

Santarpia L, Calin GA, Adam L, Ye L, Fusco A, Giunti S, Thaller C, Paladini L, Zhang X, Jimenez C, et al. 2013 A miRNA signature associated with human metastatic medullary thyroid carcinoma. Endocrine-Related Cancer 20 809-823. (https://doi.org/10.1530/ERC13-0357)

Schwarzenbach H, Nishida N, Calin GA \& Pantel K 2014 Clinical relevance of circulating cell-free microRNAs in cancer. Nature Reviews Clinical Oncology 11 145-156. (https://doi.org/10.1038/ nrclinonc.2014.5)

Schwarzenbach H, Da Silva AM, Calin GA \& Pantel K 2015 Data normalization strategies for microRNA quantification. Clinical Chemistry 61 1333-1342. (https://doi.org/10.1373/ clinchem.2015.239459)

Spitschak A, Meier C, Kowtharapu B, Engelmann D \& Putzer BM 2017 MiR-182 promotes cancer invasion by linking RET oncogene activated NF-kappaB to loss of the HES1/Notch1 regulatory circuit. Molecular Cancer 16 24. (https://doi.org/10.1186/s12943-016-0563-x) 
Toiyama Y, Takahashi M, Hur K, Nagasaka T, Tanaka K, Inoue Y, Kusunoki M, Boland CR \& Goel A 2013 Serum miR-21 as a diagnostic and prognostic biomarker in colorectal cancer. Journal of the National Cancer Institute 105 849-859. (https://doi.org/10.1093/jnci/djt101)

Wells SA Jr, Asa SL, Dralle H, Elisei R, Evans DB, Gagel RF, Lee N, Machens A, Moley JF, Pacini F, et al. 2015 Revised American Thyroid Association guidelines for the management of medullary thyroid carcinoma. Thyroid 25 567-610. (https://doi.org/10.1089/ thy.2014.0335)
Werner RA, Schmid JS, Muegge DO, Luckerath K, Higuchi T, Hanscheid H, Grelle I, Reiners C, Herrmann K, Buck AK, et al. 2015 Prognostic value of serum tumor markers in medullary thyroid cancer patients undergoing vandetanib treatment. Medicine 94 e2016. (https://doi.org/10.1097/ MD.0000000000002016)

Yan JW, Lin JS \& He XX 2014 The emerging role of miR-375 in cancer. International Journal of Cancer 135 1011-1018. (https://doi. org/10.1002/ijc.28563)

Received in final form 27 December2017

Accepted 3 January 2018

Accepted Preprint published online 3 January 2018
(C) 2018 Society for Endocrinology Published by Bioscientifica Ltd. Printed in Great Britain 